\title{
Direct Determination of Atmospheric Turbulence and Dispersion Parameters
}

\author{
D. SKIBIN \\ Nuclear Research Centre-Negev, Beer-Sheva, Israel \\ (Manuscript received 27 May 1971, in revised form 13 September 1971)
}

\begin{abstract}
A formula is presented for the frequency distribution function of wind direction, $P(\theta)$, having a lateral intensity of turbulence, $\sigma_{v} / \bar{u}$. A simple set-up is described which measures the relative frequency of electrical dc output voltages. An excellent approximation for $P(\theta)$ is obtained by using this set-up for the measurement of the output of a wind vane. Comparison of the theoretical and experimental measurements of $P(\theta)$ enables one to obtain $\sigma_{v} / u$. Sampling duration $t$ and averaging time $\tau$ can be arbitrarily chosen or changed, thus giving $\left(\sigma_{v}\right)_{t, \tau}$, which defines the lateral dispersion coefficient $\sigma_{y}$, according to Pasquill's theory.
\end{abstract}

\section{Introduction}

One of the problems, in case of an emergency release of pollutants to the atmosphere, is the determination of the concentration distribution function of the pollutant.

The Gaussian (normal) distribution is widely used as a convenient and good approximation to this function (Sutton, 1953; Pasquill, 1962; Byzova, 1965; Slade, 1968). Assuming the Gaussian solution, the problem is reduced to the determination of $\sigma_{y}$ and $\sigma_{z}$, which are the standard deviations of the concentration distribution in the directions $Y$ and $Z$, normal to the mean wind direction $X$ (where $Z$ is vertical).

Four main systems were developed which give expressions for $\sigma_{y}$ :

$\begin{array}{ll}\text { System } & \text { Expressionfor } \sigma_{y} \\ \text { Fickian } & \sigma_{y}=\left(2 K_{y} t\right)^{\frac{2}{2}} \\ \text { Sutton } & \sigma_{y}=\frac{1}{\sqrt{2}} C_{y} X^{\left(2-N_{y}\right) / 2} \\ \text { Cramer } & \sigma_{y}=\sigma_{\theta} X^{P} \\ \text { Pasquill } & \sigma_{y}=\left(\sigma_{v}\right)_{t, \tau} t\end{array}$

and similar expressions for $\sigma_{z}$, where $C_{y}, N_{y}, K_{y}$, and $P$ are constants determined empirically according to the atmospheric stability, where $\sigma_{\theta}$ is the standard deviation of wind direction, and where $\left(\sigma_{v}\right)_{i, \tau}$, the standard deviation of the wind component in the $Y$ direction, $t$ the sampling duration, and $\tau$ the averaging time.

In the routine operation of nuclear facilities or in an emergency, dispersion estimates are usually performed (Haugen, 1963; Singer and Smith, 1966) based on the systems of Sutton or Cramer, or using Pasquill's stability categories (Slade, 1968). These systems are simple and quick to operate. Nevertheless, their application is empirical in the sense that the detailed micrometeorological processes which carry and disperse the pollutant are not taken into account.

The lateral intensity of turbulence, $\sigma_{v} / \bar{u}$ (where $\bar{u}$ is the mean wind speed), can be used as a micrometeorological measure for dispersion. The usual direct and inexpensive determination of $\sigma_{v} / \bar{u}$ (Swanson and Cramer, 1965) nevertheless involves a great amount of work.

The present study suggests a quick and simple procedure for the direct determination of $\left(\sigma_{v}\right)_{t, \tau} / \vec{u}$ for different $t$ and $\tau$, from which $\sigma_{y}$ can be estimated using Pasquill's method.

\section{Definitions and assumptions}

In a plane parallel to the earth's surface, let $u$ and $v$ be the wind components along and normal to the mean wind direction ( $X$ and $Y$ components), respectively. If we let $\bar{u}$ be the mean of $u$, then, by definition of the coordinate system, $\bar{v}=0$. If we then let $s$ represent the instantaneous wind speed and $\theta$ the wind direction measured from the $X$ axis, then (Fig. 1)

$$
\begin{aligned}
s^{2} & =u^{2}+v^{2}, \\
v & =u \tan \theta .
\end{aligned}
$$

We will limit the discussion to the case where

$$
|\theta|<\frac{\pi}{2}
$$

The only cases where this condition does not apply are those of very light winds. From (3) it is clear that

$$
u>0 \text {. }
$$




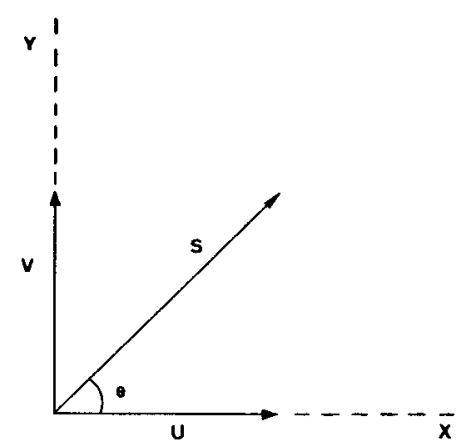

FIG. 1. Components of the wind in a plane parallel to the earth's surface.

The normal (Gaussian) distribution is considered to be the best representation of the probability distribution function of the wind components (Hesselberg and Bjorkdal, 1929; Best, 1935; Batchelor, 1961). Using this assumption, we will represent the functions for $u$ and $v$, respectively, by

$$
\begin{aligned}
& P(u) d u=\frac{\exp \left[-(u-\bar{u})^{2} /\left(2 \sigma_{u}{ }^{2}\right)\right]}{(2 \pi)^{\frac{1}{2}} \sigma_{u}} d u, \\
& P(v) d v=\frac{\exp \left[-v^{2} /\left(2 \sigma_{v}{ }^{2}\right)\right]}{(2 \pi)^{\frac{3}{2}} \sigma_{v}} d v .
\end{aligned}
$$

Isotropic turbulence is the simplest type of turbulence. However, because of the existence of the earth's surface and its topography, full isotropic turbulence cannot develop in the lowest layers of the atmosphere. Nevertheless, Hinze (1959) studied this type of turbulence extensively, because of its relative simplicity and because it is fundamental to the understanding of the phenomenon of turbulence. The concept of local isotropy further justifies the investigation of isotropic turbulence, and its use as a first approximation to the actual state of the atmosphere.

In the present work we will not assume isotropy, but will consider homogeneous and stationary turbulence, neglecting the effect of topography, so that we can assume that a certain amount of horizontal symmetry exists.

According to Sutton (1953, p. 65) "there is no reason to associate either gusts or lulls with a tendency of the wind to swing in any particular direction, and most likely $\overline{u v}=0 . "$

Under the conditions we have assumed, this reasoning holds true (Lumley and Panofsky, 1964) and like Frenkiel (1951) we will assume to a first approximation that

$$
\overline{u v}=0
$$

neglecting the influence of topography and other mechanical disturbances.

\section{Derivation of the probability distribution function of wind direction, $P(\theta)$}

There are a number of formulas which give the probability distribution function of wind direction and speed (Frenkiel, 1951; Syono and Tanaka, 1968), although they are rather complicated. Using the assumptions specified above, a simple and convenient formula for $P(\theta)$ is developed, in which one of the parameters is $\sigma_{v} / \bar{u}$. Measurement of $P(\theta)$ will readily give $\sigma_{v} / \bar{u}$.

Keeping $u$ constant, we have from Eq. (2) that

$$
(d v)_{u=\mathrm{constant}}=\left(u / \cos ^{2} \theta\right) d \theta .
$$

Using (5) and (6) and assuming (7), the probability that the wind direction will be between $\theta$ and $\theta+d \theta$ is given by

$$
\begin{aligned}
P(\theta) d \theta=\frac{1}{2 \pi \sigma_{u} \sigma_{v}} \int_{0}^{\infty} d u\{ & \exp \left[-\frac{(u-\bar{u})^{2}}{2 \sigma_{u}{ }^{2}}\right] \\
& \left.\times \exp \left[-\frac{u^{2} \tan ^{2} \theta}{2 \sigma_{v}{ }^{2}}\right] \frac{u d \theta}{\cos ^{2} \theta}\right\} .
\end{aligned}
$$

Assuming a symmetrical distribution of $u$ around $\bar{u}$, Eq. (4) means that $|u-\bar{u}|<\bar{u}$. The interpretation of this condition under the normal distribution hypothesis is that the number of cases where $|u-\bar{u}|>\bar{u}$ is negligibly small, i.e., $\bar{u}>2 \sigma_{u}$ (since more than $95 \%$ of the normal distribution is contained within a distance of $2 \sigma$ from the mean). Therefore, we can integrate (9) from $-\infty$ without any significant error, so that

$$
\begin{gathered}
P(\theta) d \theta=\frac{\exp \left[-\bar{u}^{2} /\left(2 \sigma_{u}^{2}\right)\right]}{2 \pi \sigma_{u} \sigma_{v} \cos ^{2} \theta} d \theta \int_{-\infty}^{\infty} u \\
\quad \times \exp \left[\frac{u \bar{u}}{\sigma_{u}{ }^{2}}-\left(\frac{1}{2 \sigma_{u}{ }^{2}}+\frac{\tan ^{2} \theta}{2 \sigma_{v}{ }^{2}}\right) u^{2}\right] d u .
\end{gathered}
$$

If we define

$$
X=u-\left[\bar{u} \sigma_{v}{ }^{2} /\left(\sigma_{v}{ }^{2}+\sigma_{u}{ }^{2} \tan ^{2} \theta\right)\right],
$$

we can change the variable and obtain

$$
\begin{aligned}
& P(\theta) d \theta=\frac{\exp \left\{-\left(\bar{u}^{2} \tan ^{2} \theta\right) /\left[2\left(\sigma_{v}{ }^{2}+\sigma_{u}{ }^{2} \tan ^{2} \theta\right)\right]\right\}}{2 \pi \sigma_{u} \sigma_{v} \cos ^{2} \theta} d \theta \\
& \times\left[\int_{-\infty}^{\infty} X e^{-A x^{2}} d x+\frac{\bar{u} \sigma_{v}{ }^{2}}{\sigma_{v}{ }^{2}+\sigma_{u}{ }^{2} \tan ^{2} \theta} \int_{-\infty}^{\infty} e^{-A} x^{2} d x\right],
\end{aligned}
$$

where

$$
A \equiv \frac{\sigma_{v}^{2}+\sigma_{u}^{2} \tan ^{2} \theta}{2 \sigma_{u}^{2} \sigma_{v}^{2}}
$$

The first integral in (12) vanishes because of symmetry, and since

$$
\int_{-\infty}^{\infty} e^{-A} \mathfrak{X}^{2} d \mathfrak{X}=\left(\pi A^{-1}\right)^{\frac{1}{2}}
$$


we have

$$
\begin{aligned}
& P(\theta) d \theta=\frac{\bar{u} \sigma_{v}{ }^{2} \cos \theta}{(2 \pi)^{\frac{1}{2}}} \\
& \times \frac{\exp \left\{-\left(\bar{u}^{2} \sin ^{2} \theta\right) /\left[2\left(\sigma_{v}^{2} \cos ^{2} \theta+\sigma_{u}^{2} \sin ^{2} \theta\right)\right]\right\}}{\left(\sigma_{v}^{2} \cos ^{2} \theta+\sigma_{u}^{2} \sin ^{2} \theta\right)^{3}} .
\end{aligned}
$$

Denoting the intensities of turbulence along the axes by

we have

$$
\left.\begin{array}{l}
G_{x}=\sigma_{u} / \bar{u} \\
G_{y}=\sigma_{v} / \bar{u}
\end{array}\right\}
$$

$$
\begin{aligned}
& P(\theta) d \theta=\frac{G_{y}{ }^{2} \cos \theta}{(2 \pi)^{\frac{1}{2}}} \\
& \times \frac{\exp \left\{-\sin ^{2} \theta /\left[2\left(G_{y}{ }^{2} \cos ^{2} \theta+G_{x}{ }^{2} \sin ^{2} \theta\right)\right]\right\}}{\left(G_{y}{ }^{2} \cos ^{2} \theta+G_{x}{ }^{2} \sin ^{2} \theta\right)^{\frac{3}{3}}} d \theta .
\end{aligned}
$$

It can be shown that (17) is already normalized so that $P(\theta)$ represents a probability distribution function.

\section{The system}

The set-up consists of a wind vane, a chopper and a multi-channel analyzer (Asculai, 1965). The dc voltage is the output of the wind vane and is proportional to the wind direction. The chopper (Azaria and Asculai, 1966) samples this voltage at a frequency of up to 100 Hz. The output of the chopper is a standard shaped pulse whose height is proportional to the input voltage, i.e., the wind direction. The multi-channel analyzer checks the pulses according to their heights, counts them, and stores the information in the different channels. If the direction is sampled on a $360^{\circ}$ basis, then the voltage stored in the tenth channel, for instance, is proportional to the number of times in which the wind direction was $010^{\circ}$, etc. The graph shown on the multi-channel analyzer scope is a step function, $P_{1}(\theta)$, which represents the relative frequency distribution function of wind direction. For a sampling rate as high as $100 \mathrm{~Hz}$, and considering the response time limitations of the vane (Beckman and Whitley, model 1567), $P_{1}(\theta)$ is a good approximation of $P(\theta)$. Fig. 2 is a typical $P_{1}(\theta)$ graph. Usually, for a short sampling duration, $P_{1}(\theta)$ is a symmetrical curve with a maximum in the middle, so that the most frequent wind direction is also the mean.

If we let $N / N_{0}$ be the relative frequency of the mean wind direction (where $N_{0}$ is the total number of samples in each experiment) and $\theta_{0}$ be the width of a channel (in radians), then

$$
P_{1}\left(|\theta|<\theta_{0} / 2\right)=N / N_{0},
$$

where $\theta$ is measured from the mean wind direction. On the other hand, from (17) we see that at about $\theta \approx 0$,

$$
P(\theta)_{\theta \simeq_{0}}=1 /\left[(2 \pi)^{\frac{1}{2}} G_{y}\right]
$$

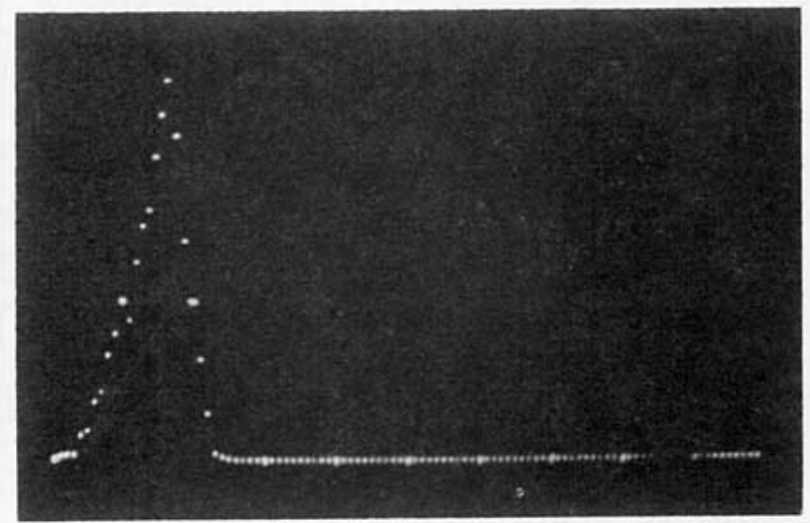

FIG. 2. Typical $P_{1}(\theta)$ graph shown on the scope of the multichannel analyzer.

so that the probability that $|\theta|<\theta_{0} / 2$ for small enough $\theta_{0}$ is given by

$$
P\left(|\theta|<\theta_{0} / 2\right)=\theta_{0} /\left[(2 \pi)^{\frac{1}{2}} G_{y}\right] .
$$

Equating (18) and (20), we have

$$
N / N_{0}=\theta_{0} /\left[(2 \pi)^{\frac{1}{2}} G_{y}\right] \text {, }
$$

or

$$
G_{y}=\theta_{0} N_{0} /\left[(2 \pi)^{\frac{1}{2}} N\right],
$$

which shows, for constant $\theta_{0}$ and $N_{0}$, that the larger the value of $N$, the steeper and narrower $P_{1}(\theta)$ becomes; this is a definition of low-intensity turbulence.

\section{Results and discussion}

At about $\theta \approx 0$, we have 1) $\sin \theta \approx \theta \ll 1,2) \cos \theta \approx 1$, and 3) $\sin \theta \ll \cos \theta$; and in this case $\sigma \approx G_{y}=\sigma_{v} / \vec{u}$. Denoting the standard deviation of the wind direction by $\sigma_{\theta}$ [equivalent to Cramer's (1965) $\left.\sigma_{A}\right]$, it is clear that at about $\theta \approx 0, P(\theta)$ behaves like the Gaussian distribution, and $\sigma_{\theta}=\sigma_{v} / \bar{u}$.

The Gaussian type of behavior is especially pronounced when $G_{x}=G_{y}$, i.e., when unstable conditions exist (Swanson and Cramer, 1965). Then

$$
P(\theta)=\frac{\cos \theta}{(2 \pi)^{\frac{1}{2}} G_{y}} \exp \left(-\frac{\sin ^{2} \theta}{2 G_{y}^{2}}\right) .
$$

In this case, even up to $\theta=15^{\circ}$, the difference between $\theta$ and $\sin \theta$ does not exceed $1.5 \%$, and the difference between $\cos \theta$ and 1 does not exceed $3.5 \%$; therefore, the behavior of $P(\theta)$ is still nearly Gaussian.

Using a table from Slade (1968, p. 102), which relates Pasquill's (1962) stability categories to $\sigma_{\theta}$, we can say that $P(\theta)$ behaves like the Gaussian distribution (denoted by $P_{N}$ ) up to $\theta \approx 15^{\circ}$ for stability categories lower than $\mathrm{D}$ in the cases where $G_{x}=G_{y}$. This can be seen in Fig. 3 where $P(\theta)$ and $P_{N}$ fit Pasquill's stabilities $\mathrm{A}, \mathrm{B}$ and $\mathrm{C}$.

According to Swanson and Cramer (1965), $G_{x} \neq G_{y}$ under neutral or stable conditions. Fig. 4 gives $P(\theta)$ and $P_{N}$ for stability categories $C, D, E$ and $F$ in the case where $\sigma_{v} / \sigma_{u}=0.5$. The difference between $P(\theta)$ and 


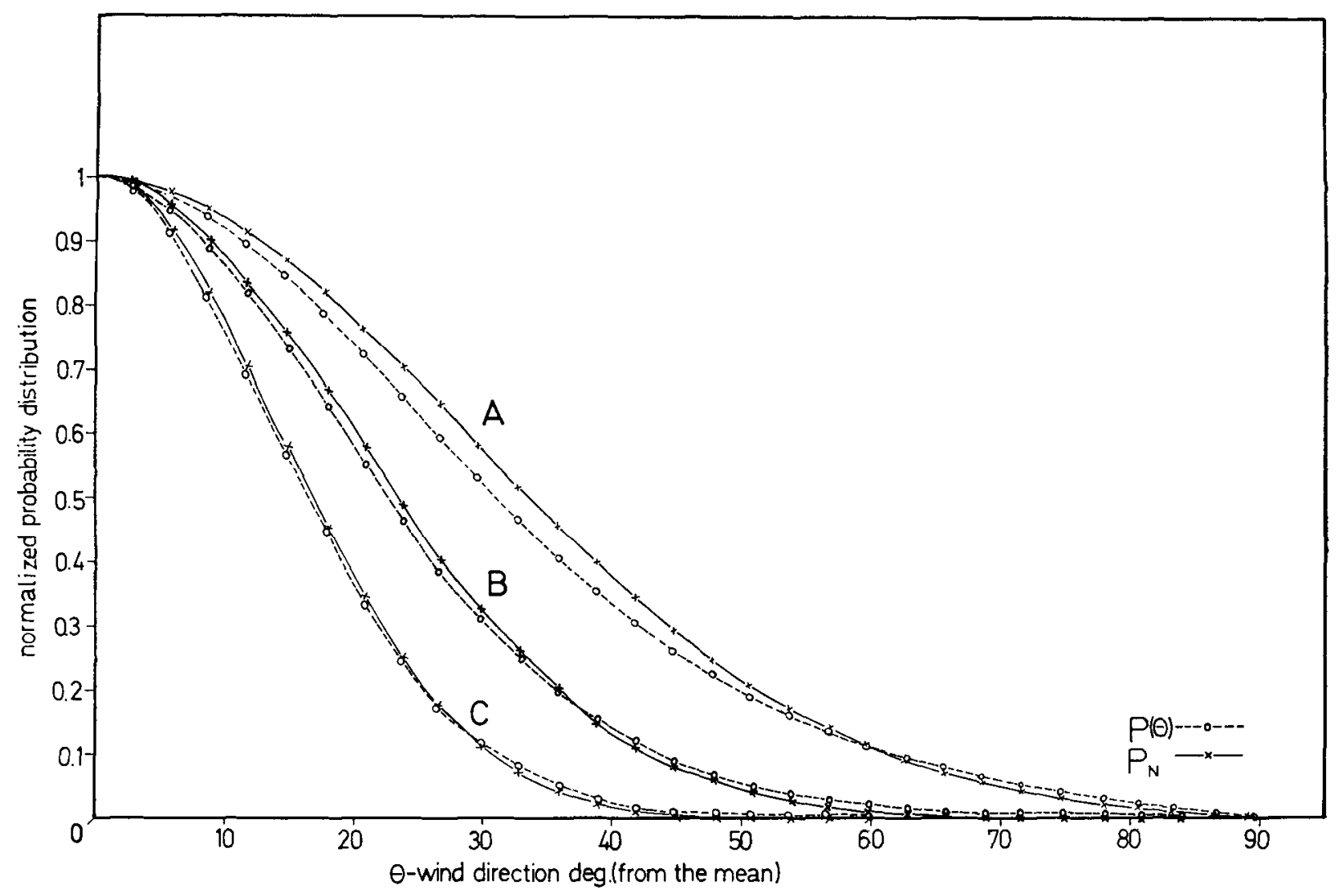

FIG. 3. Distribution of $P(\theta)$ and $P_{N}$ for the isotropic case: stability A, $G_{x}=G_{y}=0.5$; stability B, $G_{x}=G_{y}=0.35$; stability C, $G_{x}=G_{y}=0.25$.

$P_{N}$ increases as the instability increases for stable cases. The difference is due primarily to the fact that $G_{x} \neq G_{y}$, while for the unstable cases it is a result of $\theta$ deviating markedly from zero.

Five experiments were conducted to check the agreement of (17) to the experimental results. The curves of $P_{1}(\theta)$ were fitted to those of $P_{N}$ and $P(\theta)$, using the method of least squares in a CDC 3600 computer. The sum of squares in each fit is denoted by $Q$. The $Q$ resulting from the fit of $P(\theta)$ was always less than that obtained from the fit of $P_{N}$, that is, $P(\theta)$ in our experiments is a better fit to the experimental curve $\left[P_{1}(\theta)\right]$ than $P_{N}$. The relative improvement in $Q$ is given in Table 1. It is seen that $P(\theta)$ fits the data better than $P_{N}$, by $16 \%$ on the average.

TABLE 1. Relative improvement in the fit of $P(\theta)$ to experimental data as compared to the fit of $P_{N}$.

\begin{tabular}{ccccccc}
\hline $\begin{array}{l}\text { Exper- } \\
\text { iment } \\
\text { number }\end{array}$ & $\begin{array}{c}\text { Pasquill } \\
\text { stability } \\
\text { category }\end{array}$ & $G_{x}$ & $G_{\nu}$ & (rad) $^{\sigma_{\theta}}$ & (deg) & $\begin{array}{c}\text { Relative } \\
\text { improvement } \\
\text { in } Q \\
\text { (percent) }\end{array}$ \\
\hline 1 & $\mathrm{~B}$ & 0.309 & 0.107 & 0.106 & 6.05 & 5.1 \\
2 & $\mathrm{D}$ & 0.356 & 0.170 & 0.169 & 9.7 & 10.9 \\
3 & $\mathrm{AB}$ & 0.403 & 0.151 & 0.147 & 8.39 & 14.4 \\
4 & $\mathrm{AB}$ & 0.852 & 0.229 & 0.167 & 9.55 & 36.0 \\
5 & $\mathrm{C}$ & 0.414 & 0.109 & 0.103 & 5.88 & 15.4 \\
\hline
\end{tabular}

Smoothing was attempted using the overlapping interval method. This reduced the values of $Q$ by $20 \%$ but improved the fit of $P(\theta)$ relative to $P_{N}$ by more than $20 \%$ on the average.

Strong objections can be raised in this case against the use of the least-squares method. The reason is that this is a mathematical procedure whose results, $G_{x}$ and $G_{y}$, are not necessarily the real $G_{x}$ and $G_{y}$ present at the time of the experiment. This question is further raised since in the few experiments conducted, the ratio $G_{x} / G_{y}$ was as high as 4 , as compared to the results of Swanson and Cramer (1965), who obtained ratios of the order of magnitude of 2 . The problem can probably be solved by using a computer to compare the results of experiments with the proposed system, with wind components sampled simultaneously.

In order to check the agreement of $P(\theta)$ to the data, the correlation coefficient between $P(\theta)$ and the data was computed using the formula (Brooks and Carruthers, 1953, p. 303):

$$
R^{2}=1-\frac{\Sigma \epsilon_{i}^{2}}{\Sigma y_{i}}
$$

where $\epsilon_{i}=Y_{i}-E\left(y_{i}\right)$ and $y_{i}=Y_{i}-\bar{Y} ; Y_{i}$ are the experimental data, $\left[P_{1}(\theta)\right]$ and $\bar{Y}$ their mean, and $E\left(Y_{i}\right)$ the expected values, i.e., $P(\theta)$. 


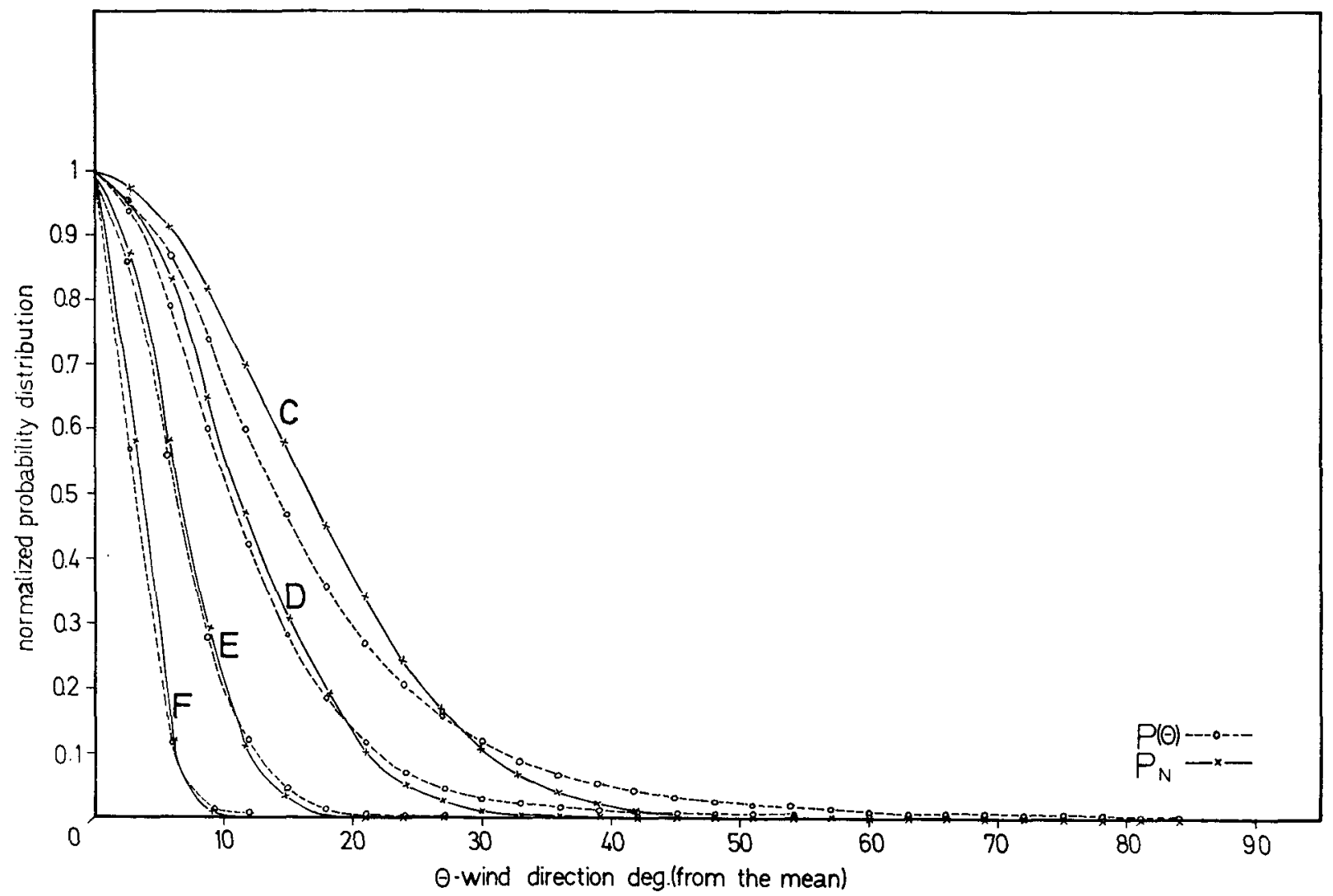

FIG. 4. Distribution of $P(\theta)$ and $P_{N}$ for the anisotropic case: stability $\mathrm{C}, G_{x}=0.5, G_{\nu}=0.25$; stability $\mathrm{D}, G_{x}=0.34$, $G_{y}=0.17$; stability E, $G_{x}=0.2, G_{y}=0.1 ;$ stability F, $G_{x}=0.1, G_{y}=0.05$.

In all the experiments the correlation coefficient $R$ exceeded 0.97 , which leads to the conclusion that $P(\theta)$ is a good fit to the experimental data.

\section{Summary and conclusions}

A new formula for the frequency distribution function of wind direction, $P(\theta)$, has been introduced. Good agreement with experimental results was reached.

Comparison of $P(\theta)$ with data obtained from the proposed chopper, multi-channel analyzer system gives direct readings of the lateral intensity of turbulence, $G_{y}=\sigma_{v} / \bar{u}$, for different sampling and averaging times.

The determination of $G_{y}$ is quick and simple and can be used as a basis for a routine assessment of dispersion coefficients, using Pasquill's method.

Acknowledgments. I am indebted to Prof. J. Neuman, head of the Department of Meteorology in the Hebrew University of Jerusalem, for his continuous help and encouragement.

\section{REFERENCES}

Asculai, E., 1965: A method of rapid determination of atmospheric diffusion parameters. U. S. Atomic Energy Commission, Rept. CONF-650407, Intern. Symp. Fission Product Release and Transport under Accident Conditions, Oak Ridge, Tenn. Azaria, H., and E. Ascuali, 1966: Chopper. Israel AEC Atomic Energy Commission, Rept. NRCN-182.

Batchelor, G. K., 1961 : The Theory of Homogeneous Turbulence. Cambridge University Press, $199 \mathrm{pp}$.
Best, A. C., 1935: Transfer of heat and momentum in the lowest layers of the atmosphere. Geophys. Mem., No. 65, Meteor. Office, London.

Brooks, C. E. P., and N. Carruthers, 1953: Handbook of Statistical Methods in Meteorology. London, Meteor Office.

Byzova, N. L., 1965: Investigation of the bottom 300-meter layer of the atmosphere. Translated by the Israel Program for Scientific Translations, Jerusalem.

Frenkiel, F. N., 1951: Frequency distribution of velocities in turbulent flow. J. Meteor., 8, 316-320.

Haugen, D. A., 1963: A simplified method for automatic computations of turbulent wind direction statistics. J. A ppl. Meteor., 2, 306-309.

Hesselberg, T., and E. Bjorkdal, 1929: Uber das Verteilungesetz der Windruhe. Beitr. Phys. Atmos., 15, 121-129.

Hinze, O. J., 1959: Turbulence. New York, McGraw-Hill, 586 pp.

Lumley, J. L., and H. A. Panofsky, 1964: The Structure of Atmospheric Turbulence. New York, Interscience, $239 \mathrm{pp}$.

Pasquill, F., 1962: Atmospheric Diffusion. London, Van Nostrand, $297 \mathrm{pp}$.

Singer, I. A., and M. E. Smith, 1966: Atmospheric dispersion at BNL. Air Water Pollution, 10, 125-135.

Slade, D. H., 1968: Meteorology and Atomic Energy. U. S. Atomic Energy Commission, Rept. TID-24190.

Sutton, O. G., 1953: Micrometeorology. New York, McGraw-Hill, $333 \mathrm{pp}$.

Swanson, R. N., and Cramer, H. E., 1965: A study of lateral and longitudinal intensities of turbulence. J. Appl. Meteor., 4, 409-417.

Syono, S., and H. Tanaka, 1966: On the frequency distribution of wind speed and direction in turbulent fow. J. Meteor. Soc, Japan, 44, 89-100. 